\title{
Metformina Minimiza as Alterações \\ Morfométricas no Músculo Sóleo de Ratos \\ Submetidos à Imobilização Articular
}

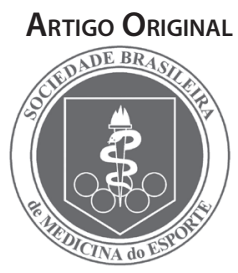

\author{
Metformin Minimizes the Morphometric Alterations in the Soleus \\ Muscle of Rats Submitted to Articular Immobilization
}

\author{
Paula Lima Bosi' \\ Gabriel Borges Delfino² \\ João Luiz Quagliotti Durigan 3 \\ Karina Maria Cancelliero ${ }^{4}$ \\ Maria Luiza Ozores Polacow ${ }^{5}$ \\ Carlos Alberto da Silva ${ }^{5}$ \\ 1. Mestranda em Fisioterapia UFSCar \\ 2. Mestrando Fisioterapia UFSCar \\ 3. Doutorando em Ciências \\ Fisiológicas UFSCar \\ 4. Doutoranda em Fisioterapia \\ - UFSCar \\ 5. Prof (a). Dr(a). PPG - Mestrado em \\ Fisioterapia - UNIMEP
}

\section{Endereço para correspondência: \\ Paula Lima Bosi \\ Rua Sete de Setembro no 1320, \\ apto. 112 \\ Bairro: Centro \\ São Carlos - SP- CEP: 13560-180 \\ E-mail: plbosi@terra.com.br}

Submetido em 07/11/2007

Versão final recebida em 12/03/2008

Aceito em 11/04/2008

\section{RESUMO}

A proposta deste trabalho foi avaliar o músculo sóleo (S) de ratos submetidos à imobilização articular por sete dias, associado ou não ao tratamento com metformina (MET, 1,4mg. $\left.\mathrm{ml}^{-1}\right)$ por meio de análises morfométricas. Ratos adultos Wistar $(n=5)$ foram divididos nos grupos: controle $(C)$, imobilizado em posição neutra do tornozelo (I), tratado com metformina (M), imobilizado tratado com metformina (I + MET). Foram avaliadas a área das fibras, a densidade de área do tecido conjuntivo intramuscular e a massa muscular do S. A análise estatística foi realizada pelo teste de normalidade, ANOVA e de Tukey $(p<0,05)$. A imobilização reduziu o peso muscular (mg) do S (34\%). No grupo M não houve alteração significativa do peso muscular quando comparado com o grupo C. Já no grupo I + MET foi observado aumento do peso muscular em 29,6\% quando comparado com o grupo I. O tratamento com metformina não alterou a área da fibra muscular quando comparado com grupo C. Já no grupo I, houve redução de $44 \%$ na área da fibra. Com relação ao grupo I + MET, houve aumento de 22\% quando comparado com o grupo I. Por outro lado, ao compararmos o grupo C com o grupo I + MET, houve redução de 31\%. Ao avaliar a densidade de área do tecido conjuntivo, observou-se que o grupo I apresentou elevação de $216 \%$ quando comparado com o grupo C. No grupo I + MET, houve redução de $67 \%$ comparado com o grupo I. O tratamento com metformina em músculos submetidos à imobilização minimizou a redução da área das fibras do $\mathrm{S}$, bem como o aumento do tecido conjuntivo. Esses resultados sugerem que a metformina pode favorecer recuperação mais rápida na fase pós-imobilização.

Palavras-chave: atrofia, desuso, metformina, reabilitação..

\section{ABSTRACT}

The aim of this study was to evaluate the effect of metformin treatment on the muscle mass, fibers area and connective tissue area density in soleus (S) muscle under articular immobilization. Methods and Results: Male Wistar rats (250-300g) were divided in 4 groups $(n=5)$ : control, treated with metformin, immobilized and immobilized treated with metformin. Immobilization was performed by acrylic resin orthoses on the left hindlimb keeping the ankle in neutral position during 7 days. The animals were euthanatized and the $\mathrm{S}$ muscle was dissected and weighed. Samples of its ventral portion were treated for inclusion in paraffin and stained in Hematoxylin-Eosin (H:E). The results were obtained through analyses of the muscular fiber area (images analyzed - Image Pró-plus 4,0), as well as of intramuscular connective tissue by means of planimetry. The statistical analysis was performed by normality test followed by ANOVA and Tukey $(p<0.05)$. Hindlimb immobilization during 7 days promoted significant reduction $(p<0.05)$ of $35 \%$ in the muscular mass; $44 \%(p<0.05)$ in the fiber area and increase of $216 \%(p<0.05)$ in the intramuscular connective tissue. The metformin in immobilized group promoted significant alterations $(p<0.05)$ in the muscular mass. In addiction, it was observed significant increase $(p<0.05)$ of $29.6 \%$ in the fibers area and significant reduction $(p<0.05)$ of $67 \%$ in the intramuscular connective tissue. Conclusion: The metformin treatment minimized the reduction of muscle fibers area, as well as the increase of connective tissue in hindlimb immobilized muscle. These results suggest that metformin should be used to increase fast muscle recovery after hindlimb immobilization.

Keywords: disuse atrophy, metformin, rehabilitation. 


\section{INTRODUÇÃO}

O desuso muscular induz uma gama de efeitos deletérios, tais como a proliferação do tecido conjuntivo intramuscular, hipotrofia muscular, redução das reservas de glicogênio, da densidade dos capilares, dos sarcômeros em série, da força e resistência à fadiga (1).

No intuito de minimizar os eventos desencadeados pelo desuso muscular, diversas técnicas têm sido utilizadas, com destaque para estimulação elétrica neuromuscular, fármacos como o clembuterol e suplementos como o sulfato de vanadil, a creatina, a glutamina e a metformina ${ }^{(2-4)}$.

A biguanida metformina tem sido utilizada como um importante agente no tratamento de diabetes do tipo II devido à sua ação antihiperglicemiante ${ }^{(5)}$. O fármaco possui ação multifatorial, por reduzir a liberação de glicose pelo fígado, e promover reduções na gliconeogênese, oxidação hepática de ácidos graxos e na absorção intestinal da glicose, além de possibilitar o aumento da captação periférica da hexose, assim como a glicogênese ${ }^{(6)}$. A ação anti-hiperglicemiante da metformina fundamenta-se na elevação da captação da glicose via tecidos periféricos, sem aumentar a secreção de insulina; no entanto, para que o efeito farmacológico seja efetivo, a insulina deve estar presente, mesmo em concentrações reduzidas. Outra característica importante é que ela não induz hipoglicemia, mesmo em indivíduos normoglicêmicos ${ }^{(7,8)}$.

Estudos prévios realizados pelo nosso grupo de pesquisa demonstraram que o tratamento com metformina promoveu melhora no metabolismo muscular da glicose, além de aumento na densidade de área de fibras musculares em ratos submetidos à desnervação neuromuscular ${ }^{(9-11)}$. Nesse contexto, recentemente Bosi et al ${ }^{(4)}$ demonstraram que a metformina promoveu a elevação nas reservas de glicogênio muscular de membros posteriores de ratos submetidos à imobilização durante sete dias.

Assim, a avaliação do tratamento com o fármaco metformina na condição de imobilização articular necessita ser estudada com mais detalhes, pelas evidências de que essa possa ser uma terapia coadjuvante no processo de reabilitação e recuperação muscular pós-imobilização. Pautado nessa observação, o objetivo do trabalho foi avaliar morfometricamente o músculo sóleo de ratos submetidos à imobilização articular, tratados ou não com metformina.

\section{MÉTODOS}

Ratos Wistar (três a quatro meses, 250-300g) foram mantidos em condições controladas de biotério, recebendo água e ração ad libitum e tratados segundo recomendações do Guide for Care Use of Laboratory Animals $^{(12)} \mathrm{e}$ aprovado pelo comitê de ética em experimentação animal da UFSCar (Processo 03/06).

Os animais foram divididos em quatro grupos experimentais com cinco animais cada $(n=5)$ : controle $(C)$, imobilizado $(I)$, tratado com metformina (M) e imobilizado tratado com metformina (I + MET).

Para a imobilização, os ratos foram anestesiados com pentobarbital sódico $(50 \mathrm{mg} / \mathrm{kg})$, tendo a pata posterior esquerda imobilizada com órtese de resina acrílica durante sete dias, a qual manteve a articulação do tornozelo em posição neutra $\left(90^{\circ}\right)$, deixando as articulações do joelho e quadril livres ${ }^{(13)}$.

A metformina foi diluída na água disponível durante sete dias para os animais na concentração de $1.4 \mu \mathrm{g} \cdot \mathrm{ml}^{-1}$, realizando-se a troca do frasco de âmbar a cada 48 horas $^{(9)}$.
Após o período experimental, foram avaliadas: massa muscular (mg), área das fibras e densidade de área do tecido conjuntivo intramuscular.

Para a análise morfométrica, o segmento ventral do músculo sóleo foi fixado em solução tamponada de formol a 10\% e processado em parafina, obtendo-se vários cortes transversais não seriados de $7 \mu \mathrm{m}$ de espessura, que foram corados pela hematoxilina-eosina (HE).

Foi utilizado o sistema de análise de imagens constituído do software Image Pró-plus 4.0 (Media Cybernects), câmera digital (JVC) acoplada a um microscópio (Zeiss) integrado ao microcomputador. Todas as imagens foram captadas com objetiva de 10x.

As áreas de 375 fibras do músculo sóleo, por animal, foram assim obtidas: 15 fibras por campo, sendo cinco campos por corte, num total de cinco cortes por animal. Utilizou-se de um retículo quadriculado para delimitar o campo e a escolha das 15 fibras foi feita, aleatoriamente, coincidindo com as interseções de retas.

Para a análise da densidade de área do tecido conjuntivo intramuscular foi utilizado o sistema de planimetria por contagem de pontos ${ }^{(14)}$, sendo a quantificação realizada por um retículo com quadrados de $2.500 \mu^{2}$ contendo 56 intersecções de reta. Foram contados os pontos coincidentes no endomísio e perímisio, em cinco áreas por corte, sendo cinco cortes por animal, perfazendo um total de 1.400 pontos por animal. A área relativa do tecido conjuntivo (densidade de área) foi calculada dividindo-se a soma do número de pontos coincidentes nas intersecções de reta sobre o tecido conjuntivo (endomísio e perimísio) pelo número total de pontos.

A análise estatística foi realizada inicialmente pelo teste de normalidade de Kolmogorov-Smirnov e pelo teste de homocedasticidade (critério de Bartlett). Como os dados se apresentaram normais, foi aplicada a ANOVA one way seguida do teste de Tukey; em todos os cálculos foi fixado o nível crítico de 5\% ( $p<0,05)$.

\section{RESULTADOS}

A imobilização reduziu significativamente $(p<0,05)$ o peso muscular (mg) do S em 34\% (média \pm dpm, C: 123,5 $\pm 2,1 \mathrm{mg} \times$ l: 81,3 \pm 1,89mg), a área da fibra em 44\% (média \pm dpm, C: $2.575 \pm 560 \mu \mathrm{m}^{2} \times \mathrm{l}$ : $1.438 \pm 352 \mu \mathrm{m}^{2}$ ) e elevou a densidade de área do tecido em 216\% (C: $8,82 \pm 3,5 \%$ X : $27,92 \pm 5,4 \%$ ) (tabela1, figura 1).

O tratamento com metformina (grupo M) não alterou a massa, a área, nem o conteúdo de tecido conjuntivo do músculo sóleo (média $\pm \mathrm{dpm}, \mathrm{C:}: 123,5 \pm 2,1 \mathrm{mg} \times \mathrm{M}: 134,5 \pm 3,89 \mathrm{mg} ; \mathrm{C}: 2.575 \pm 560 \mu \mathrm{m}^{2} \times \mathrm{M}$ : $\left.2,689 \pm 468 \mu \mathrm{m}^{2} ; \mathrm{C}: 8,82 \pm 3,5 \% \times \mathrm{M}: 11,08 \pm 5,5 \%\right)$.

Tabela 1. Média \pm dpm da massa do músculo sóleo (mg), área das fibras musculares $\left(\mu \mathrm{m}^{2}\right)$, bem como densidade de área do tecido conjuntivo (\%), dos grupos controle (C), imobilizado (I), tratado com metformina (M) e imobilizado tratado com metformina $(I+M)$

\begin{tabular}{c|c|c|c|c}
\hline & $\mathbf{C}$ & $\mathbf{M}$ & $\mathbf{I}$ & $\mathbf{I}+\mathbf{M}$ \\
\hline Massa (mg) & $123,5 \pm 2,1$ & $134,5 \pm 3,89$ & $81,3 \pm 1,89^{*} \#$ & $105,4 \pm 7,53^{*}+\#$ \\
\hline Área $\left(\boldsymbol{\mu m ^ { 2 }}\right)$ & $2.575 \pm 560$ & $2.689 \pm 468$ & $1.438 \pm 352^{*} \#$ & $1.761 \pm 494^{*}+\#$ \\
\hline Conjuntivo (\%) & $8,82 \pm 3,5$ & $11,08 \pm 5,52$ & $27,92 \pm 5,36^{*} \#$ & $18,81 \pm 4,72^{*}+\#$ \\
\hline
\end{tabular}

$\mathrm{N}=5, \mathrm{p}<0,05,{ }^{*}$ comparado com o grupo $\mathrm{C}$, \# comparado com o grupo CMET, † comparado com o grupo I. 

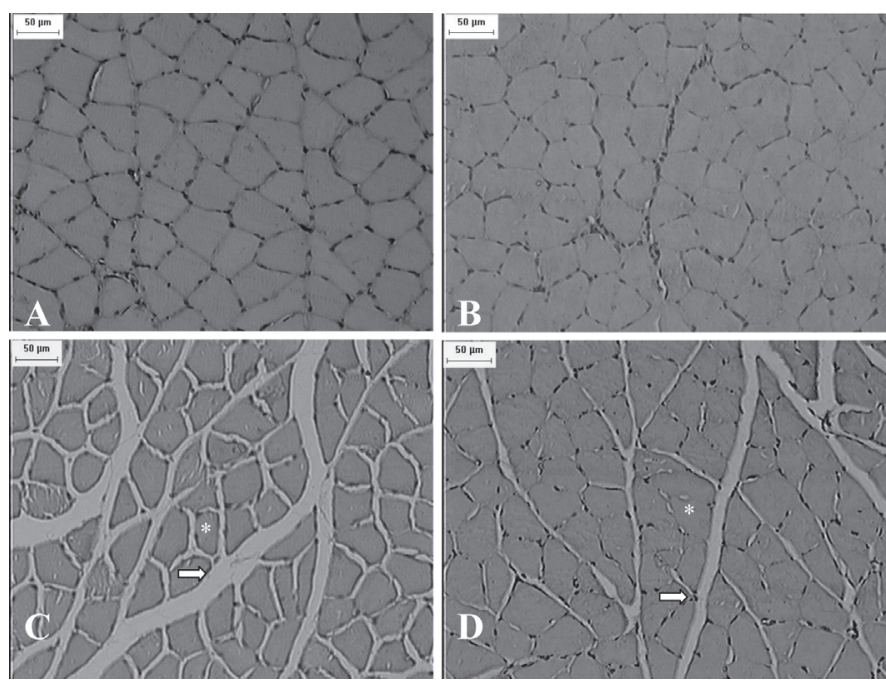

Figura 1. A) Imagem das fibras do músculo sóleo (hematoxilina-eosina - HE). Grupo controle. B) Músculo sóleo controle tratado com metformina. C) Músculo sóleo imobilizado em posição neutra do tornozelo sete dias. Observar a redução da área das fibras musculares (asterisco) e aumento do tecido conjuntivo (seta) em relação ao grupo controle. D) Músculo sóleo imobilizado em posição neutra do tornozelo sete dias e tratado com metformina. Observar aumento da área das fibras musculares (asterisco) e redução do tecido conjuntivo (seta) em relação ao grupo imobilizado (aumento, 10x)

O tratamento com metformina no músculo imobilizado foi eficaz em reduzir a área do tecido conjuntivo em 67\% (I: 27,92 \pm 5,4\% x I + MET: 18,81 \pm 4,7\%), em elevar o peso muscular em 29,6\% e a área da fibra em 22\% (média \pm dpm, I: 81,3 \pm 1,89mg x I + M: 105,4 \pm 7,53mg; l: $1.438 \pm 352 \mu \mathrm{m}^{2} \times \mathrm{I}+\mathrm{M}: 1.761 \pm 494 \mu \mathrm{m}^{2}$ ).

\section{DISCUSSÃO}

A imobilização articular é comumente observada quando o membro é mantido em gesso ou órtese após lesão ortopédica, o que leva a rápida diminuição do peso muscular, indicando a necessidade da intervenção fisioterapêutica para sua reabilitação(15)

Nesse sentido, Kannus et al. ${ }^{(16)}$ relataram redução de 69\% da área das fibras do músculo sóleo imobilizado em posição encurtada por aparelho gessado durante três semanas. Neste trabalho a imobilização em posição neutra do tornozelo promoveu redução significativa de 35\% na massa muscular, bem como de 44\% na área das fibras musculares no período de sete dias, resultados semelhantes aos estudos anteriores.

Além disso, foi encontrado o valor médio de $2.574,88 \mu \mathrm{m}^{2}$ para o músculo sóleo do grupo controle, estando de acordo com os valores de referência apresentados pela literatura, que podem variar de 2.084 a $3.500 \mu m^{(17-19)}$

Com relação à área relativa do tecido conjuntivo intramuscular de músculos normais, a literatura apresenta valores de 1 a 10\%, dependendo do músculo avaliado ${ }^{(19,20)}$. Neste estudo observou-se valor de $8,82 \%$ de área relativa do tecido conjuntivo no músculo sóleo do grupo controle, o que demonstra estar dentro dos valores de referência citados.

A imobilização durante sete dias promoveu aumento significativo de $216 \%$ na área do tecido conjuntivo se comparado com o grupo controle. Resultado corroborativo com Józsa et al. ${ }^{(21)}$, que demonstraram que independentemente do modelo de desuso muscular estudado (imobilização, tenotomia ou denervação), a quantidade de teci- do conjuntivo no endomísio e perimísio aumenta significativamente, desde $50 \%$ a $700 \%$

Infelizmente ainda não há tratamento eficaz para prevenir as alterações fisiológicas inerentes ao desuso muscular. Porém, com a identificação de algumas vias de sinalização relacionadas à hipotrofia muscular e com o avanço da indústria farmacêutica, possivelmente será viável o desenvolvimento de fármacos no intuito de minimizar os efeitos deletérios promovidos pelo desuso muscular, ou mesmo a associação desses a recursos fisioterapêuticos ${ }^{(15)}$.

Nesse contexto, a metformina potencializa a ação da insulina, previne o desenvolvimento de resistência à insulina, favorece a síntese de glicogênio em diferentes tecidos, podendo ser uma ferramenta farmacológica de ação anti-catabólica no músculo esquelético (22-24).

Trabalhos anteriores desenvolvidos pelo nosso grupo de pesquisa demonstraram o efeito da metformina na elevação das reservas de glicogênio dos músculos periféricos submetidos à imobilização $\operatorname{articular}^{(4)}$. Além disso, foi demonstrado que o fármaco minimizou a redução da massa muscular e da densidade da área das fibras no músculo sóleo desnervado em ratos ${ }^{(10)}$. Desse modo, esses resultados corroboram os apresentados neste estudo, já que a metformina também minimizou a redução do peso e área das fibras do músculo sóleo submetidos à imobilização articular, destacando que tanto a desnervação ou imobilização são modelos clássicos de desuso muscular.

Esses resultados são atribuídos ao fato de a metformina aumentar a sensibilidade do receptor insulínico, desencadeando, dentre vários efeitos, o aumento da síntese protéica. Gore et al.(25) estudaram pacientes com resposta hipermetabólica à lesão severa hiperglicêmica e verificaram o efeito anabólico da metformina, fato significativo, já que nesses pacientes observa-se a perda de massa muscular inerente ao catabolismo protéico. As possibilidades apresentadas para explicar tal efeito no músculo residem, por exemplo, na inibição da gliconeogênese via diminuição da liberação de alanina (aminoácido envolvido na gliconeogênese), que na forma liberada agrega proteína muscular. Portanto, a diminuição do seu efluxo estaria perpetuando um efeito anticatabólico. Outra vertente para o efeito anticatabólico da metformina seria o deslocamento da forma de substrato utilizado para a manutenção da homeostasia muscular, da quebra de aminoácidos como combustível à utilização da glicose que se encontra disponibilizada na periferia.

Os resultados deste trabalho demonstraram que a metformina minimizou a redução da massa muscular, bem como da área das fibras no músculo sóleo durante sete dias de imobilização. Desse modo, esses resultados assinalam uma importante ação anticatabólica da metformina, fato de extrema importância para os profissionais da reabilitação no período e pós-período de imobilização articular.

Os dados referentes à densidade de área do tecido conjuntivo apresentados neste estudo demonstram que, com a metformina, o aumento do tecido conjuntivo no músculo sóleo durante a imobilização articular foi bem menor do que no músculo imobilizado sem tratamento. Tal fato sugere que o equilíbrio entre a formação do colágeno e a atividade das colagenases tem forte relação com a sensibilidade tecidual à insulina, uma vez que modificações na composição da matriz extracelular têm sido demonstradas no músculo insulino-resistente ${ }^{(26)}$

Nesse sentido, foi demonstrado que a metformina exerce atividade inibitória das metaloproteinases; com isso, pode estar descompensando a relação síntese/degradação. O processo de síntese 
de colágeno é dependente do IGF-1, que se apresenta reduzido no músculo imobilizado. Esses fatores associados podem estar limitando a formação do colágeno e indiretamente protegendo a fibra muscular imobilizada $(27,28)$.

Esse achado é extremamente importante, porém, são necessários mais estudos para determinar o mecanismo pelo qual a metformina minimizou o aumento da densidade de área do tecido conjuntivo no músculo imobilizado durante sete dias, já que tais alterações determinam a elasticidade, rigidez e extensibilidade muscular.

\section{CONCLUSÃO}

O tratamento com metformina durante sete dias minimizou a redução da massa muscular, área das fibras e aumento da densidade de área do tecido conjuntivo no músculo sóleo submetido à imobilização articular, demonstrando ação anticatabólica, fatores esses que podem favorecer uma reabilitação acelerada na fase pós-imobilização. Os resultados deste estudo apontam para intervenção farmacológica como coadjuvante durante a reabilitação, no intuito de minimizar os efeitos deletérios do desuso muscular.

Destaca-se ainda que a metformina vem sendo utilizada para diversos fins terapêuticos, porém, considerações devem ser feitas sobre a escassez de trabalhos que utilizem o fármaco com o objetivo de minimizar os efeitos deletérios inerentes ao desuso muscular. Nesse sentido, há necessidade de maior número de estudos, com análises diversificadas que auxiliem na definição dessa importante ação farmacológica.

\section{AGRADECIMENTOS}

Agradecemos à Fapesp pelo apoio financeiro via bolsa de iniciação científica. (Processo: 05/60094-2).

Todos os autores declararam não haver qualquer potencial conflito de interesses referente a este artigo.

\section{REFERÊNCIAS BIBLIOGRÁFICAS}

1. Reardon KA, Davis J, Kapsa RM, Choong P, Byrne E. Myostatin, insulin-like growth factor-1, and leukemia inhibitory factor are upregulated in chronic human disuse muscle atrophy. Muscle Nerve 2001;24:893-9.

2. Cancelliero KM. Estimulação elétrica neuromuscular associada ao clembuterol melhora o perfi metabólico muscular de membro imobilizado de ratos. Tese de mestrado, PPG Fisioterapia, UNIMEP, 2004

3. Delfino GB, Cancelliero KM, Durigan JLQ, Silva CA. Efeito do sulfato de vanadil sobre o comprometimento metabólico. Rev Bras Med Esporte 2006;12:356-60.

4. Bosi PL, Delfino GB, Durigan JLQ, Cancelliero KM, Silva CA. Pharmacological protection with metformin in the muscle metabolic alteration inherent of the functional limitation. Brazilian Archives of Biology and Technology. 2008;51(3)

5. Bolen S, Feldman L, Vassy J, Wilson L, Yeh HC, Marinopoulos S, et al. Systematic review: comparative effectiveness and safety of oral medications for type 2 diabetes mellitus. Ann Intern Med 2007;147:386-99.

6. Staels B. Metformin and pioglitazone: effectively treating insulin resistance. Curr Med Res Opin 2006;22 Suppl 2:S27-37

7. DeFronzo RA, Barzilai N, Simonson DC. Mechanism of metformin action in obese and lean noninsulindependent diabetic subjects. J Clin Endocrinol Metab 1991;73:1294-301.

8. Bailey CJ. Hypoglycaemic and anti-hyperglycaemic drugs for the control of diabetes. Proc Nutr Soc 1991;50:619-30

9. Silva CA, Guirro RJ, Polacow MLO, Silva HC, Tanno AP, Rodrigues D. Efeito da metformina e eletroestimulação sobre as reservas de glicogênio do músculo sóleo normal e desnervado. Revista Brasileira de Fisioterapia 1999:3:55-60.

10. Polacow MLO, Silva CA, Guirro RJ, Campos MR, Borges JP. Estudo morfométrico do músculo sóleo desnervado de ratos tratados pela associação de metformina e estimulação elétrica. Revista Brasileira de Fisioterapia 2003;7:57-64

11. Guirro RRJ, Silva CA, Forti F, Cancelliero KM. Análise do músculo esquelético desnervado tratado com metformina e/ou estimulação elétrica de baixa freqüência. Revista Brasileira de Fisioterapia 2004;8:21-7.

12. National Research Council. Guide for the Care and Use of Laboratory Animals. Washington, DC, USA: National Academy Press, 1996

13. Silva CA, Guirro RRJ, Polacow MLO, Cancelliero KM, Durigan JLQ. Proposal for rat hindlimb joint immobilization: orthosis with acrylic resin model. Braz J Med Biol Res 2006:39:979-85.
14. Mathieu O, Cruz-orive LM, Hoppeler H, Weibel ER. Measuring error and sampling variation in stereology: comparison of the efficiency of various methods for planar image analysis. J Microsc 1981;121:75-88.

15. Glass DJ. Signalling pathways that mediate skeletal muscle hypertrophy and atrophy. Nat Cell Biol 2003;5:87-90.

16. Kannus $P$, Jozsa $L$, Jarvinen $T L$, Kvist $M$, Vieno $T$, Jarvinen $T A$, et al. Free mobilization and low- to highintensity exercise in immobilization-induced muscle atrophy. J Appl Physiol 1998;84:1418-24.

17. Jones SW, Roger JH, Philip AK, Barbara O, Nicholas P, Paul LG. Disuse atrophy and exercise rehabilitation in humans profoundly affects the expression of genes associated with the regulation of skeletal muscle mass. Journal of the Federation of American Societies for Experimental Biology. 2004;18:1025-7.

18. Gomes ARS, Coutinho EL, França CN, Polonio J, Salvini TF. Effect of one stretch a week applied to the immobilized soleus muscle on rat muscle fiber morphology. Braz J Med Biol Res 2004;37:1473-80.

19. Delp MD, Duan C. Composition and size of type I, IIA, IID/X, and IIB fibers and citrate synthase activity if rat muscle. J Appl Physiol 1996;26:261-70.

20. Nicks DK, Beneke WM, Key RM, Timson BF. Muscle fibre size and number following immobilization atrophy. J Anat 1989;163:1-5.

21. Józsa L, Kannus P, Thoring J, Reffy A, Jarvinen M, Kvist M. The effect of tenotomy and immobilization on intramuscular connective tissue. J Bone Joint Surg 1990;72:293-7.

22. Bailey CJ, Turner RC. Metformin. N Engl J Med 1996;29:574-9.

23. Matthaei S, Stumvoll M, Kellerer M, Haring HU. Pathophysiology and pharmacological treatment of insulin resistance. Endocr Rev 2000;21:585-618.

24. Ma TC, Buescher JL, Oatis B, Funk JÁ, Nash AJ, Carrier RL, et al. Metformin therapy in transgenic mouse model of Huntington's disease. Neurosci Lett 2006;14:312-26.

25. Gore DC, Wolf SE, Sanford A, Herndon DN, Wolfe RR. Influence of metformin on glucose intolerance and muscle catabolism following severe burn injury. Ann Surg 2005;241:334-42.

26. Berria R, Wang L, Richardson DK, Finlavson J, Belfort R, Mandaarino L. Increased collagen content in insulin-resistant skeletal muscle. Am J Physiol 2006;290:560-5.

27. Froesch ER, Schmid C, Schwander J, Zapf J. Action of insulin like growth factors. Ann Rev Physiol 1995;47:443-67.

28. Li L, Mamputu JC, Wiernsperger N, Renier G. Signaling pathways involved in human vascular smooth muscle cell proliferation and matrix metalloproteinase-2 expression: inhibitory effect of metformin. Diabetes 2005:54:2227-34 